\title{
Corela
}

Cognition, représentation, langage

HS-35 | 2022

Anaphore et pronoms en anglais : convergences, différences et complémentarité de quelques approches linguistiques

\section{Perspective phonologique sur les pronoms}

\section{Stephan Wilhelm}

\section{(2) OpenEdition}

\section{Journals}

Édition électronique

URL : https://journals.openedition.org/corela/14404

DOI : $10.4000 /$ corela. 14404

ISSN : 1638-573X

Éditeur

Cercle linguistique du Centre et de I'Ouest - CerLICO

Référence électronique

Stephan Wilhelm, «Perspective phonologique sur les pronoms », Corela [En ligne], HS-35 | 2022, mis en ligne le 04 janvier 2022, consulté le 26 janvier 2022. URL : http://journals.openedition.org/corela/ 14404 ; DOI : https://doi.org/10.4000/corela.14404

Ce document a été généré automatiquement le 26 janvier 2022.

\section{(c) (1)(2)}

Corela - cognition, représentation, langage est mis à disposition selon les termes de la licence Creative Commons Attribution - Pas d'Utilisation Commerciale - Partage dans les Mêmes Conditions 4.0 International. 


\title{
Perspective phonologique sur les pronoms
}

\author{
Stephan Wilhelm
}

\section{Introduction}

1 Le Trésor de la Langue Française informatisé ( $\mathrm{TLFi}^{1}$ ) définit le pronom comme un « [m]ot qui a la propriété de remplir dans une phrase les mêmes fonctions que le nom ou le syntagme nominal et qui désigne directement quelqu'un ou quelque chose (déictique) ou représente un segment du discours (anaphorique).»

2 Nous reviendrons sur certains aspects importants de cette définition au cours de cette étude. Il convient de souligner à ce point que si cette vulgarisation s'avère utile pour définir une catégorie particulièrement difficile à circonscrire (cf. les articles de Puckica et de Cotte dans ce volume), elle demande cependant à être modifiée en partie. Affirmer qu'un pronom anaphorique représenterait (ou « reprendrait») un segment du discours est en effet inexact (cf. les notes du colloque sur l'anaphore). C'est pourquoi il parait plus juste de souligner que le recours aux pronoms permet «d'assurer une certaine économie dans le discours, en évitant les répétitions et en fondant leur interprétation sur le contexte et la situation » (cf. texte de présentation du colloque TLD6 ${ }^{2}$ ).

3 On reconnait traditionnellement l'existence de plusieurs catégories de pronoms :

1) les pronoms personnels, appelés ainsi parce qu'ils portent la marque de la personne (première, deuxième ou troisième personne) ;

2) les pronoms réfléchis, qui représentent, souvent en fonction de complément, l'entité déjà nommée qui se trouve également être le sujet grammatical ;

3) les pronoms démonstratifs, qui constituent une classe particulière de déictiques ;

4) les pronoms interrogatifs, qui permettent de s'enquérir de l'identité de leur référent ;

5) les pronoms indéfinis, qui ne désignent personne ou rien en particulier ;

6) les pronoms relatifs, qui permettent de relier une proposition relative à un « antécédent » présent dans le contexte ; 
7) le « pronom » there, qui constitue un cas particulier en anglais.

Le corpus sur à partir duquel nous travaillerons comprend 10 segments :3

(1) what I do ... [is] coach people (1. 5, 00'24")

(2) What is it that drives you in your life today (1.13,00'56")

(3) a) All of us here have great minds / b) we all know how to think (ll. 16-17, 00'56")

(4) With our minds we can rationalize anything (1. 18, 00'56")

(5) when emotion comes into it, the wiring changes (1. 21, 01'23")

(6) especially those who are very smart (1.23, 1'23")

(7) that person's ability to ... do something beyond themselves (1. 44, 02'52")

(8) whatever it is for you (1. 49, 02'52")

(9) the real question, which is $\left(1.56,03^{\prime} 43^{\prime \prime}\right)$

(10) But there was emotion that was there (11. 102-103, 06'39")

5 Il comprend au moins une occurrence de chacune des catégories de pronoms recensées plus haut, ce qui nous permettra d'avoir une vision assez large de la phonologie des pronoms :

\begin{tabular}{|l|l|}
\hline \multicolumn{1}{|c|}{ Segment } & \multicolumn{1}{c|}{ Description des occurrences } \\
\hline (1) what I do & Pronom relatif « fusionné » \\
\hline (2) What is it that & $\begin{array}{l}\text { Pronom interrogatif / personnel / relatif } \\
\text { (interrogative clivée) }\end{array}$ \\
\hline $\begin{array}{l}\text { (3) } \text { a. } \text { All of us here have great minds } \\
\text { b. we all }\end{array}$ & Pronoms personnels + quantifieur flottant \\
\hline (4) anything & Pronom indéfini \\
\hline (5) it & Pronom personnel de genre neutre \\
\hline$(6)$ those & Pronom démonstratif \\
\hline (7) themselves & Pronom personnel réfléchi \\
\hline (8) whatever & Pronom relatif « fusionné » \\
\hline (9) which & Pronom relatif \\
\hline$(10)$ there $\ldots$ there & « Pronom » there \\
\hline
\end{tabular}

Tableau 1 : Description des occurrences présentes dans le corpus

6 Pour aborder la phonologie des pronoms, nous nous proposons de prendre appui sur deux phénomènes phonologiques majeurs, fortement liés entre eux, mais distincts, qui affectent les pronoms au même titre que la plupart des mots dits "grammaticaux ", à savoir la réduction - c'est-à-dire l'existence de formes phonologiquement faibles - et l'accentuation des pronoms dans les énoncés.

7 Nous examinerons tout d'abord la manière dont réduction et accentuation s'appliquent à chacune des grandes catégories de pronoms recensés plus haut et émettrons quelques observations au fil de l'eau, puis nous proposerons une synthèse des phénomènes observés. Cette synthèse nous permettra de mettre en évidence certains des liens qui existent entre forme phonologique et valeur sémantique des pronoms et de formuler quelques hypothèses plus générales.

\section{Formes fortes et formes faibles}

8 Afin de bien appréhender la nature des formes faibles, qui résultent du phénomène de réduction, il convient de garder à l'esprit le fait que l'anglais est une langue à rythme 
accentuel (en anglais, stress-timed language) par opposition au français, langue à rythme dit syllabique.

9 En français, toutes les syllabes sont accentuées sensiblement au même niveau, comme dans l'énoncé suivant :

[1] Sij'a-vais su, je luị au-raịs dịt.

10 En anglais, par contraste, seules certaines syllabes sont accentuées :

[2] If I had known, I would have told him.

11 Ces syllabes accentuées sont produites à une fréquence ordinairement perçue comme régulière, phénomène désigné sous le nom d'isochronie. ${ }^{4}$ L'alternance entre syllabes accentuées et syllabes non accentuées n'est pas sans incidence sur la prononciation des mots de l'anglais. Au niveau lexical, par exemple, la voyelle de la seconde syllabe du mot banana, accentuée, conserve toute sa "couleur phonétique ", pour employer une métaphore synesthésique, alors que les voyelles des $1^{\text {ère }}$ et $3^{\mathrm{e}}$ syllabes, non accentuées, se réduisent jusqu'à devenir des schwas. Il peut même arriver que certaines voyelles disparaissent tout à fait pour laisser place à des consonnes syllabiques.

12 Les conséquences du rythme syllabique de l'anglais sont également observées au niveau des énoncés. Dans la chaîne parlée, les voyelles des mots accentués conservent en effet leur couleur phonétique alors que celles des mots non accentués se réduisent. Les voyelles les plus fréquemment entendues dans ces mots sont alors le schwa ou les voyelles de кIT ou de FOOT $^{5}$. On observe également des élisions consonantiques et des consonnes syllabiques. [2] peut ainsi être réalisé comme /'If aI d 'nəひn a wd əv 'təold $\mathrm{Im} /$. La forme réduite de ces mots non accentués tend à se systématiser et à être répertoriée dans certains dictionnaires.

13 Les mots de l'anglais qui conservent leur forme pleine sont les mots lexicaux (content words), souvent définis comme des mots dont le «sens » est lexical, alors que ceux qui ont des formes réduites sont les mots grammaticaux (function words), définis comme ayant un « sens » grammatical. Bien qu'il soit relativement aisé de comprendre à quoi renvoient ces définitions, il s'avère plus problématique d'expliquer précisément ce en quoi consistent ces « sens » lexicaux et grammaticaux.

Nous proposons ici de définir les mots lexicaux comme des signifiants permettant de se référer directement à des entités extralinguistiques, c'est-à-dire des entités du monde réel, que celles-ci soient concrètes ou abstraites. Plus précisément, il s'agit de signifiants qui désignent des propriétés et, pour ce qui est des verbes et des substantifs, renvoient à des notions - c'est-à-dire des ensembles de propriétés pouvant immédiatement être appréhendées par des locuteurs partageant un même arrière-plan linguistique et culturel. Le fait de prononcer le mot «chat», par exemple, active immédiatement une représentation des propriétés "petit animal domestique », "très doux ", « qui mange des souris ", " qui ronronne », etc. , alors que le fait de prononcer les pronoms relatifs qui ou que ne produit rien de tel. On pourrait certes également désigner un chat en employant le pronom « lui ». Dans ce cas, cependant, la désignation ne s'opérerait pas directement, mais par le truchement d'opérations mentales - en l'occurrence, celles qui sous-tendent le fonctionnement de l'anaphore. C'est le propre des mots grammaticaux de marquer de telles opérations.

15 Au même titre que les prépositions, les conjonctions, les déterminants et les auxiliaires, les pronoms font partie des mots grammaticaux, et la plupart possèdent en 
conséquence des formes réduites. Nous nous proposons à présent d'examiner la réduction de chaque grande catégorie de pronoms recensée plus haut.

\subsection{Les pronoms personnels}

16 Nous nous concentrerons essentiellement sur les formes sujet et complément de ces pronoms, soit :I/me, you, he / him - she / her - it, we/us, you, they/them.

\subsubsection{Le pronom personnel singulier de 1 ère personne $I$}

17 La forme forte du pronom personnel singulier de $1^{\text {ère }}$ personne est /aI/. On en observe quelques occurrences dans l'enregistrement, par exemple I'm not exaggerating (00'21", absent du script, mais visible dans le sous-titrage). Contrairement à ce que suggèrent certains ouvrages de phonologie, il existe également des formes faibles de ce pronom : / $\mathrm{a} /, / \Lambda /$ et $/ \partial / .^{6}$ On en trouve au moins une occurrence dans l'enregistrement $(1.8 \mathrm{du}$ script, 00'36") : the bottom line of why I'm here.

\subsubsection{Le pronom personnel de $2^{\mathrm{e}}$ personne you}

18 La forme forte du pronom personnel de deuxième personne personne est /ju:/. On observe dans l'enregistrement des occurrences proches de cette forme, par exemple à la 1.6: You didn't learn it by just learning principles (00'24"). Les formes faibles de you sont $/ \mathrm{ju} /, / \mathrm{jv} /$ et $/ \mathrm{j} ə / .^{7}$ De telles occurrences sont observées dans l'enregistrement : 'cos you got in it (1.7, 00'24"), Thank you - "Thankya » (1. 1, 00'13") et What do they tell you? «tell ya » (1. 78, 05'00"). ${ }^{8}$

19 Ces deux derniers énoncés, dans lesquels une forme faible est observée alors que you se trouve être le dernier mot de l'énoncé, illustrent le fait que les pronoms personnels sont les seuls mots grammaticaux susceptibles d'apparaitre sous leur forme faible en position finale (cf. Cruttenden $2014: 275$, Roach 2009 : 88-89).

\subsubsection{Les pronoms personnels singuliers de $3^{\mathrm{e}}$ personne de genre masculin et féminin}

Les formes fortes des pronoms personnels singuliers de troisième personne de genre masculin et féminin sont respectivement /hi:/ et / $\mathrm{Si}$ /. Leurs formes faibles attestées sont /hi/, /i/ et /Si/ (Wells 2008, Roach 2009, Cruttenden 2014). Ces formes soulèvent au passage la question des voyelles dites "neutralisées ", dont la brièveté s'approche de celle de la voyelle de kit et la couleur phonétique s'approche fréquemment de celle de la voyelle fermée de fleece. Dès les années 1960, ce phénomène de neutralisation était observé par Gimson (1965), qui proposait de transcrire la forme faible de she "/SI/ ([Si])». Au début des années 2000, Cruttenden (2001) proposait des transcriptions apparentées pour les deux pronoms de troisième personne he et she: «/hi/, /i/, /I/ ([hi])» et «/SI/ ([Si])». Pour le pronom de genre masculin, on note que la réduction s'opère aussi parfois sur le plan consonantique (élision de $/ \mathrm{h} /$ ). 


\subsubsection{Le pronom pluriel de $1^{\mathrm{e}}$ personne we}

21 La forme forte du pronom pluriel de première personne est /wi:/, et sa forme faible / wi/ (parfois transcrite /wI/). Le corpus comporte une occurrence de la forme faible de ce pronom : (3b) we all know how to think (l. 17, 00'56").

Dans des contextes non emphatiques (cf. note 22 pour notre définition de l'emphase), il est parfois difficile de distinguer la forme faible de la forme forte de ce pronom. L'enregistrement comprend ainsi un passage dans lequel la longueur de la voyelle de we pourrait évoquer une forme forte: when we /wi:/ watched the debate (1. 98, 06'39"), mais aussi une répétition du même énoncé dans laquelle tout trait de longueur contrastive semble avoir disparu: when we /wi/ watched the debate... (1. 98, 06'39"). Dans les faits, la frontière phonétique entre forme forte et forme faible est parfois ténue.

\subsubsection{Le pronom pluriel de $3^{\mathrm{e}}$ personne they}

Il s'agit du seul pronom personnel à ne pas avoir de forme faible. La raison paraît en être que ce pronom comporte une diphtongue, type vocalique qui tend à résister à la réduction'. Nous proposerons plus loin une autre explication qui, sans être contradictoire, pourrait être complémentaire (cf. synthèse et discussion).

\subsection{Les pronoms réfléchis}

24 A la troisième personne, ces pronoms polysyllabiques sont formés par l'adjonction de la terminaison -self/selves aux formes complément des pronoms personnels de troisième personne. Aux première et deuxième personnes, cette terminaison est ajoutée aux formes qui dérivent de leur génitif historique :

[3] a. myself /mat'self/ (mI-, mə-)

b. yourself /jo: 'self/ ou /juə 'self/ - (jə-)

c. himself / hIm 'self/ (Im-)

c'. herself /h3: 'self/ (hə-, 3:-, ə-)

c". itself /It'self /

d. ourselves / (,)a汸 'selvz/ (a:-)

e. yourselves /jo:'self/ ou /jøə 'self/, /jə- /

f. themselves /ðəm 'selvz/

Tous ces pronoms possèdent des variantes dans lesquelles l'élément de gauche apparait sous sa forme faible (cf., dans le corpus, (7) that person's ability to ...do something beyond themselves, ce qui s'explique d'autant plus facilement que les groupes de deux syllabes accentuées successives tendent à être évités en anglais. Dans ces pronoms polysyllabiques accentués sur la deuxième syllabe, on comprend aisément que la première syllabe se réduise.

\subsection{Les pronoms indéfinis}

Il s'agit également de polysyllabes. Ils sont formés au moyen de déterminants quantifieurs (some, any, every, no) auquel on adjoint le morphème -thing pour se référer à des inanimés, ou les morphèmes -one ou -body pour se référer à des animés humains. ${ }^{10}$ La règle veut que ces composés soient accentués sur le premier élément. Ceci explique 
qu'ils n'aient pas normalement de forme faible. ${ }^{11}$ Le corpus contient une occurrence de pronom indéfini : (4) With our minds we can rationalize anything (1.18 - 00'56").

\subsection{Les pronoms démonstratifs}

27 Le cas des pronoms démonstratifs, dont le corpus renferme une occurrence ((6) especially those who are very smart [1.23, 01'21"]), présente un intérêt particulier. Ces pronoms ne possèdent en effet pas de forme faible. ${ }^{12}$ L'explication que fournit Cruttenden (2014: 270) de ce phénomène est en outre propre à nous interpeller: " [S]ome words are predisposed by their function in language to be accented ${ }^{13}$. These lexical words are typically main verbs, adverbs, nouns, adjectives and demonstrative pronouns" (c'est nous qui soulignons). Cruttenden assimile en effet les pronoms démonstratifs à des mots lexicaux alors qu'il s'agit de mots grammaticaux qui marquent des opérations mentales et non de signifiants associés à des notions (cf. introduction).

Pour tenter de rendre compte du comportement des pronoms démonstratifs, nous suggérerons que ceux-ci possèdent un trait commun avec les mots lexicaux, à savoir qu'ils permettent, comme eux, de désigner directement des entités extralinguistiques. La différence est que les mots lexicaux le font, en tant que signifiants associés à des ensembles de propriétés alors que les pronoms démonstratifs le font par deixis (cf. la définition du TLFI fournie en introduction). Les pronoms démonstratifs portent en outre la marque du nombre, ce qui les distingue de tous les autres pronoms. Il s'agit là d'un autre trait qui les rapproche des substantifs.

\subsection{Les pronoms relatifs}

\subsubsection{THAT}

Les deux occurrences de THAT relatif présentes dans le corpus consistent en des formes très faibles. La voyelle, considérablement réduite, a presque totalement disparu pour laisser place à une consonne syllabique :

(2) What is it that drives you in your life today (1. 13,00'56")

(10) But there was emotion that was there.

30 Il est ici tentant de rendre compte de ce haut degré de réduction en postulant l'existence d'une corrélation entre poids phonologique et masse sémantique. La légèreté de la forme phonologique de THAT pourrait en effet s'expliquer ici par son peu de valeur sémantique, voire référentielle. (La référentialité de ce THAT relatif est d'ailleurs si faible que certains supposent qu'il pourrait s'agir non d'un pronom, mais d'un simple relateur, voir d'un "complémenteur ", au même titre que THAT conjonctif [cf. par exemple Auwera 1985]).

\subsection{Les pronoms relatifs « fusionnés »}

31 Ceci est d'autant plus remarquable lorsque l'on compare ce relatif aux pronoms relatifs appelés « fusionnés ", dont on trouve deux occurrences dans le corpus :

(1) What I do...

(8) Whether it be your business, your contribution to society, money - whatever it is for you. 
De tels pronoms n'ont en effet pas de forme faible, et ce contraste semble apte à corroborer l'hypothèse de la correspondance entre poids phonologique et charge sémantique, les relatifs « fusionnés » ayant incontestablement un poids référentiel plus important que le relatif тHAT. Dans la mesure où ils «contiennent leur propre antécédent ", ils désignent en effet des entités extralinguistiques de manière directe (on pourrait d'ailleurs gloser WHAT par « ce que » ou « les choses que »). Ils comportent en outre la marque du genre (personne ou non personne [cf. Cotte 2012]).

\subsection{Les pronoms interrogatifs}

Le corpus contient une occurrence de WHAT pronom interrogatif :

(2) What is it that drives you in your life today?

Les pronoms interrogatifs n'ont pas non plus de forme faible. D'une certaine manière, il pourrait sembler plus difficile de considérer ici que ce fait résulte d'une corrélation entre poids phonologique et charge sémantique. Contrairement aux relatifs fusionnés, les pronoms interrogatifs ne renvoient en effet à aucune entité précise, puisque leur trait WH-, que leur thématisation est destinée à souligner, signale avant tout une indéfinitude, voire un vide sémantique que l'on s'attend à voir comblé, souvent par l'interlocuteur. En contrepartie, les pronoms interrogatifs comportent bien la marque du genre, à l'instar des relatifs fusionnés.

\subsection{Le « pronom » there}

Il s'agit d'un cas particulier. Il n'existe d'ailleurs pas de véritable consensus sur la nature de there. Certains dictionnaires ne lui reconnaissent qu'un statut d'adverbe, quel que soit son contexte d'occurrence, alors que d'autres le décrivent dans certain cas comme un pronom à part entière. C'est le cas du dictionnaire Merriam Webster's en ligne $^{14}$, qui en fournit entre autres la définition suivante :

[4] there pronoun \'ther, sense 1 is also ther $\backslash[. .$.$] (Entry 2$ of 4 )

1 -used as a function word to introduce a sentence or clause // there shall come a

time

2 -used as an indefinite substitute for a name // hi there ${ }^{15}$

there noun \'ther $\backslash$ (Entry 3 of 4$)$

1 : that place or position // there is no here and no there ... in pure space (James Ward)

2 : that point // you take it from there ${ }^{16}$

L'occurrence présente dans le corpus, (10) But there was emotion that was there, évoque les constructions dites « existentielles» (ou "prédications d'existence ») et se trouve parfois analysée comme clivée en there ${ }^{17}$ Elle fournit quoi qu'il en soit un éclairage singulier sur la genèse des constructions existentielles. À l'origine de cet énoncé se trouve en effet la prédication Emotion was there, dans laquelle there adverbe de lieu remplit la fonction d'attribut de l'objet.

\section{Ce there est ensuite thématisé, et l'on obtient => There was emotion. ${ }^{18}$}

Une fois thématisé, there subit un processus de désémantisation qui permet de former l'énoncé => There was emotion (there), dans lequel il ne désigne plus véritablement le lieu, mais, par métonymie, la présence et par conséquent l'existence. Un deuxième there peut alors apparaître en position finale sans que l'énoncé soit mal formé ou 
véritablement redondant. À la manière d'un pronom personnel, there permet alors la formation de question tags : There was emotion, wasn't there?

Si l'on reçoit l'analyse de constructions comme (10) But there was emotion that was there en tant que clivées en there, il est également possible de mettre en lumière une deuxième similitude entre ce pronom locatif et les pronoms personnels, son rôle évoquant alors celui du pronom personnel de genre neutre (IT) des clivées canoniques.

Quoi qu'il en soit, de tels énoncés corroborent à nouveau l'hypothèse de la corrélation entre poids phonologique et charge sémantique. Car le poids phonologique de there se réduit par définition lors de sa désémantisation, de telle sorte que, dans les prédications d'existence, il apparaît la plupart du temps sous sa forme réduite alors que l'adverbe de lieu conserve normalement sa forme pleine. Ceci se vérifie dans le segment du corpus, dans lequel la première occurrence de there est réalisée sous sa forme réduite alors que l'adverbe there, en fin d'énoncé, l'est sous sa forme forte.

\subsection{Le cas de IT}

IT, le pronom personnel neutre de la troisième personne, n'a ni forme forte, ni forme faible attestée. Nous reviendrons cependant ici sur une occurrence déjà traitée lors du colloque sur l'anaphore en 2017. Un examen attentif des formes phonétiques de IT employées par Tony Robbins révèle que l'orateur emploie tantôt des variantes fortis ([It] / [Ir(-V)]) et tantôt des variantes lenis de ce pronom ([IP] / [Iø] ${ }^{19}$. Ces formes ne sont pas en variation libre. Dans la plupart des cas, le choix entre les deux types de variantes est conditionné par l'environnement phonétique dans lequel elles sont produites. Dans l'énoncé suivant, en revanche: Because when emotion comes into it, the wiring changes in the way it functions. (1. 21, 01'23"), une variante fortis est utilisée avant une pose alors que, dans l'enregistrement, ce sont des variantes lenis qui sont ordinairement observées dans ce contexte. Pour rendre compte de ce choix, nous suggérions qu'il convenait de prendre en considération le faible degré d'accessibilité mentale du référent de IT (cf. Ariel 2000), du fait du caractère diffus et de la complexité des éléments discursifs qui permettent de construire son antécédent. ${ }^{20}$ L'hypothèse que nous formulions était que, dans l'idiolecte du Tony Robbins, l'opposition allophonique fortis/lenis pouvait servir à marquer divers degrés distincts d'accessibilité mentale.

Nous ne développerons pas davantage ici ces considérations, mais elles seront reprises et élaborées dans la deuxième partie de cette réflexion, consacrée à l'accentuation des pronoms.

\subsection{Synthèse}

43 L'examen du corpus nous permet à ce point de dresser une liste des pronoms qui se réduisent et de ceux qui ne se réduisent pas (cf. tableau 2 infra). Les pronoms réfléchis ne sont pas pris en compte dans ce tableau dans la mesure où leur structure polysyllabique contraint dans la plupart des cas la réduction de leur élément inaccentué.

44 Les pronoms sans forme faible (pronoms démonstratifs, relatifs "fusionnés » et interrogatifs) semblent de manière générale être porteurs d'un sémantisme plus marqué - voire être caractérisés par une référentialité plus nette - que ceux qui se 
réduisent (relatif THAT, pronom there et IT si l'on considère ses variantes lenis comme relevant de la réduction). Ceci suggère qu'il existe une corrélation entre le poids phonologique d'une forme linguistique et sa charge sémantique. Les pronoms personnels de genre masculin et féminin, sans contredit référentiels ${ }^{21}$, genrés et portant la marque du nombre, semblent cependant constituer une exception à ce principe. Non seulement ils subissent ordinairement la réduction, mais ils apparaissent encore sous leur forme faible même en position finale, contrairement à tous les autres mots grammaticaux. Nous tenterons d'apporter une explication à cet état de fait en abordant la question de l'accentuation.

\begin{tabular}{|l|l|}
\hline Pronoms à forme(s) faible(s) ou réduite(s) & Pronoms sans forme faible \\
\hline - Pronoms personnels & - Pronoms démonstratifs \\
- Relatif that & - Pronoms relatifs « fusionnés » \\
- « Pronom » there dans les tournures & - Pronoms interrogatifs \\
existentielles ou les « clivées en there » & - Pronoms indéfinis \\
- it (emploi de formes lenis en alternance & \\
avec des formes fortis) & \\
\hline
\end{tabular}

Tableau 2 : Liste des pronoms du corpus qui se réduisent / ne se réduisent pas

\section{L'accentuation des pronoms}

Bien qu'intimement liée à la question des formes fortes/formes faibles, l'accentuation des pronoms constitue une problématique distincte de celle-ci. Il arrive d'ailleurs que des formes non accentuées restent fortes (ceci s'applique par exemple aux déterminants démonstratifs ou encore aux mots grammaticaux en position finale ${ }^{22}$ ). A l'inverse, il existe des cas où des syllabes accentuées se réduisent, même si ceux-ci restent marginaux. Ceci est vrai notamment de la seconde syllabe de because. ${ }^{23}$

Au niveau de l'énoncé (ou de la «phrase»), il importe de distinguer deux types d'accentuation. Il existe tout d'abord une accentuation « ordinaire » (que l'on appellera ici phrastique secondaire ${ }^{24}$ ), qui concerne la syllabe accentuée de chaque pied. Les syllabes ainsi accentuées ont été définies dans la première partie de cette réflexion par contraste avec les syllabes non accentuées de l'anglais, langue à rythme accentuel. Tous les mots lexicaux portent normalement un accent phrastique secondaire, quel que soit leur degré de proéminence dans l'énoncé. Il existe ensuite une accentuation principale, qui coïncide avec la syllabe tonique (ou nucléaire), c'est-à-dire le pivot de chaque groupe intonatif. ${ }^{25}$ L'accent tonique confère une proéminence particulière au mot considéré comme porteur de l'information la plus importante en contexte de communication.

Sauf situation particulière, c'est la syllabe accentuée du dernier mot lexical qui porte normalement l'accent tonique dans un group intonatif. Cette règle est connue en anglais sous le nom de Last lexical item (ou L.L.I.) rule. Une illustration en est fournie cidessous par la transcription intonative de l'énoncé employé dans la première partie (section 1.1.) : If he had known, he would have told him.

Hors contexte particulier, cet énoncé sera normalement produit de la manière suivante: / If I had KNOWN, / I would have TOLD him / (les syllabes toniques sont 
indiquées au moyen des majuscules, des caractères gras et du soulignement; les accents phrastiques secondaires sont simplement marqués par des majuscules).

Dans une situation de communication où l'on voudrait faire comprendre au coénonciateur que si l'on avait été mieux renseigné, on aurait parlé à un individu en particulier, et non à qui que ce soit d'autre, c'est cependant le pronom personnel désignant l'individu qui porterait l'accent tonique : / If I had KNOWN, / I would have told $\underline{\text { HIM }}$ (not anybody else) /

50 Tout mot est donc susceptible de porter un accent nucléaire, y compris les mots grammaticaux, au nombre desquels les pronoms. Lorsque le placement de l'accent tonique n'est pas déterminé par la règle du dernier élément lexical (LLI rule), on parle alors d'accent emphatique ${ }^{26}$.

51 Dans cette seconde partie, nous nous efforcerons de déterminer les règles qui président à l'accentuation des pronoms. Nous verrons quels pronoms reçoivent de manière prototypique un accent secondaire, à la manière des mots lexicaux, puis nous nous pencherons sur quelques cas particuliers d'accentuation nucléaire avant de dégager de nos observations des principes plus généraux.

\subsection{Les pronoms réfléchis}

Comme souligné supra (1.1), les pronoms réfléchis sont ordinairement des polysyllabes accentués sur le deuxième élément. A l'instar des mots lexicaux, ils reçoivent par conséquent au moins un accent phrastique secondaire.

Les pronoms réfléchis reçoivent parfois l'accent tonique. Ceci se produit lorsqu'ils apparaissent en position finale et dans les contextes emphatiques (cf. note 23). L'enregistrement contient l'occurrence suivante, où se superposent valeur contrastive et valeur expressive (cf. note 23): / Somebody who's working for you, you know, or a partner, or even yourSELF / (11. 76-77, 05'00").

Dans le segment suivant, en revanche, that person's ability to .../ do something be $\underline{\text { YOND }}$ themselves / (1. 44, 02'52"), le pronom réfléchi themselves reçoit un accent phrastique secondaire alors que la préposition qui le précède porte l'accent tonique. L'énonciateur aurait $\mathrm{pu}$ choisir de lui faire porter l'accent tonique puisque son caractère polysyllabique lui confère sur un plan rythmique un statut comparable à celui d'un mot lexical. Comme c'est parfois le cas (cf. Larreya \& Rivière 1999), c'est pourtant la préposition qui le précède qui reçoit l'accent nucléaire.

\subsection{Les pronoms indéfinis}

Comme souligné supra (1.1), les pronoms indéfinis sont eux aussi des polysyllabes. Ils sont normalement accentués sur leur premier élément et reçoivent au moins un accent phrastique secondaire. Notre corpus contient une occurrence du pronom anything ainsi accentué :

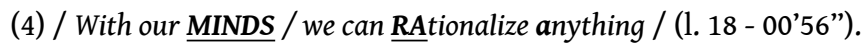

Dans la seconde unité intonative, c'est le verbe rationalize qui reçoit l'accent tonique. C'est en effet ce verbe qui exprime l'information principale de manière quasicontrastive, puisque l'énonciateur insiste ici sur la capacité de l'esprit humain à tout analyser, à la différence de l'émotion. ${ }^{27}$ 
Dans les trois énoncés qui suivent, par contraste, les pronoms indéfinis anything et anybody reçoivent un accent nucléaire emphatique :

[5] / We can make Anything happen / (1. 18, 00'56")

[6] / We can get ourselves to do Anything / (1. 104, 07'03")

[7] / Can you get through to Anybody / $\underline{\text { YES }}$ or no? / (1l. 105-106, 07'03")

Dans ces trois cas, l'énonciateur met en relief le fait que tout élément de la classe désignée par le pronom indéfini peut être concerné sans exception. Ce sens pourrait être rendu en français par l'adjonction d'un adverbe tel absolument ${ }^{28}$. Nous avons ici affaire à un renforcement sémantique par expression du haut degré. Il s'agit d'une emphase expressive telle que nous la définissions plus haut (cf. note 23).

\subsection{Les pronoms démonstratifs}

Non seulement les pronoms démonstratifs n'ont pas de forme faible, mais ils reçoivent toujours au moins un accent phrastique secondaire. Le corpus comprend une occurrence de pronom démonstratif accentué de la sorte :

(6) / especially those who are very SMART / (1. 23, 01'21").

L'accentuation systématique des pronoms démonstratifs est vraisemblablement due au fait qu'ils désignent directement des entités du monde extralinguistique, bien que n'étant pas des signifiants lexicaux. Comme suggéré supra (cf. 1.4), c'est probablement pour cette raison que Cruttenden $(2014: 270)$ les assimile aux mots lexicaux.

61 Dans la mesure où l'accentuation confère une mesure de "poids » phonologique aux formes linguistiques, cette accentuation systématique des pronoms démonstratifs paraît une fois de plus corroborer l'hypothèse de la corrélation entre substance phonologique et charge sémantique.

\subsection{Les pronoms relatifs « fusionnés »}

Les pronoms relatifs fusionnés reçoivent normalement au moins un accent phrastique secondaire. Les deux occurrences présentes dans le corpus portent respectivement un accent secondaire et un accent tonique emphatique :

(1) / What I DO... /

(8) / WHATever it is for you /

L'occurrence de whatever reçoit même, de manière non canonique, un accent sur la première syllabe alors que l'accent lexical frappe normalement le second élément de ce type de composé. Comme nous le suggérions supra (1.6), les pronoms relatifs fusionnés peuvent être considérés comme dotés d'un poids sémantique assez marqué, puisqu'ils intègrent leur propre antécédent. Ceci renforce à nouveau l'hypothèse de la correspondance entre élaboration phonologique et charge sémantique.

\subsection{Les pronoms interrogatifs}

Les mots interrogatifs (pronoms, adverbes et déterminants) reçoivent normalement un accent phrastique secondaire, mais non l'accent nucléaire. Ce fonctionnement pourrait sembler contre-intuitif dans la mesure où ce sont précisément eux qui signalent la présence du vide sémantique que la question met en évidence et qui précisent la nature de l'information sollicitée. On pourrait par conséquent s'attendre à ce qu'ils reçoivent 
eux-mêmes l'accent tonique. L'ensemble des occurrences présentes dans l'enregistrement confirment néanmoins que ce n'est pas le cas :

[8] / How did you learn LANguage? / (1. 6, 00'24")

[9] / How do you make a CHANge? / (11. 42-43, 02'52")

[10] / what is it that's shaping that person's ability ... to conTRIbute / (11. 43-44, 02'52")

[11] / How do you take what you're DREAming of / and make it HAppen? / (ll. 48-49, 02'52")

[12] / what's the difference in somebody's life if you look at somebody like those people that you've given everything TO? / (11. 56-57, 03'43")

[13] / Why didn't you aCHIEve something? / (11. 75-76, 05'00")

[14] / What's the REAson people say they failed to achieve? / (11. 77-78, 05'00")

[15] / What do they TELL you? / (1. 78, 05'00')

[16] / What do all those, including the Supreme Court, have in Common? / (1. 85, 05'47")

Dans tous ces énoncés, un accent nucléaire placé sur le mot interrogatif ne semblerait justifié qu'en contexte contrastif. On pourrait d'une certaine manière imaginer plus facilement que des pronoms interrogatifs puissent recevoir un accent nucléaire en position de sujet. Pourtant, les occurrences de WHAT présentes dans l'enregistrement ne présentent pas cette configuration. Dans des constructions clivées comme (2) / What IS it that / DRIves you in your life / to $\underline{D A Y}$ ? / et / What IS it? / (1. 65, 04'19") - qui peut être considérée comme une clivée elliptique - aussi bien que dans des questions ordinaires, telles que / What IS your motive for action? / (1.13,00'56"), c'est le verbe copulatif qui suit qui porte l'accent nucléaire et non le pronom interrogatif.

\subsection{Le « pronom » there}

Dans les prédications d'existence, le pronom there ne porte pas l'accent tonique. Il peut même ne recevoir aucune accentuation. Il apparait alors sous sa forme faible (cf. 1.8). C'est le cas dans le corpus (la première occurrence de there est une forme faible qui ne porte pas d'accent) :

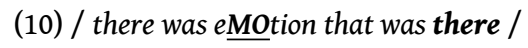

67 Il convient de noter le parallèle entre ce peu de poids phonologique et la désémantisation que le pronom there a subie (cf. 1.8).

\subsection{THAT relatif}

THAT relatif apparait normalement toujours sous sa forme faible et n'est en principe jamais accentué, même s'il est possible d'imaginer certains usages métalinguistiques, voire contrastifs, dans lesquels il pourrait recevoir un accent nucléaire emphatique ${ }^{29}$. Une fois encore, cette « discrétion " phonologique semble aller de pair avec le peu de poids sémantique qui lui est associé. Il n'est d'ailleurs pas dénué d'intérêt de noter que THAT relatif dérive étymologiquement et sémantiquement d'un démonstratif (voir par exemple Khalifa 1999 : 202-204). Son homonymie avec le pronom déictique тНАт évoque par conséquent à nouveau une manière de désémantisation.

\subsection{Les pronoms personnels}

69 A l'exception de IT et de they, tous les pronoms personnels possèdent des formes faibles. Ils ne portent jamais d'accent phrastique secondaire. En revanche, ils peuvent recevoir 
un accent nucléaire emphatique. L'enregistrement comporte deux occurrences de pronoms ainsi accentués dans un contexte contrastif :

[17] / How easy for ME / to tell HIM / what he should DO / (1. 96, 06'34").

Nous reviendrons à présent sur une occurrence déjà examinée lors du colloque sur l'anaphore. Il s'agit de / Emotion is IT / (1. 104, 07'03"), qui comporte une occurrence de IT accentué, situation considérée comme non canonique ${ }^{30}$. Pour rendre compte de l'accentuation de IT dans cet énoncé, nous suggérions qu'il convenait à nouveau de prendre en compte le signalement de l'effort à fournir pour accéder au référent en raison de la multiplicité et de la complexité des déclencheurs d'antécédent ${ }^{31}$. L'échelle de marquage d'accessibilité mentale définie par Ariel (2000 - cf. figure 1) montre en effet que l'accentuation des pronoms signale une accessibilité plus faible.

zero $<$ reflexives $<$ poor agreement markers $<$ rich agreement markers $<$ reduced/cliticized pronouns $<$ unstressed pronouns $<$ stressed pronouns $<$ stressed pronouns + gesture $<$ proximal demonstrative $(+\mathrm{NP})<$ distal demonstrative $(+\mathrm{NP})<$ proximal demonstrative $(+\mathrm{NP})$ + modifier $<$ distal demonstrative $(+\mathrm{NP})+$ modifier $<$ first name $<$ last name $<$ short definite description $<$ long definite description $<$ full name $<$ full name + modifier

Fig. 1 : Échelle de marquage d'accessibilité mentale (Ariel 2000)

\section{Synthèse et discussion}

Le recensement des pronoms généralement accentués et non accentués (cf. tableau 3) présente d'importantes similitudes avec celui des formes pronominales qui se réduisent/ne se réduisent pas. Ceci est compréhensible dans la mesure où les deux phénomènes sont liés, quoique distincts. Pour définir les principes fondamentaux mis en lumière par le comportement phonologique des pronoms, il convient cependant aussi de prendre en compte les facteurs qui président à l'accentuation emphatique des pronoms personnels.

\begin{tabular}{|l|l|}
\hline Pronoms généralement non accentués & Pronoms généralement accentués \\
\hline - Pronoms personnels (sauf en cas & - Pronoms démonstratifs \\
d'accentuation emphatique) / Pronoms & - Pronoms relatifs « fusionnés » \\
réfléchis & - Pronoms interrogatifs \\
- Relatif THAT & - Pronoms indéfinis \\
- « Pronom » there dans les tournures & \\
existentielles et apparentées & \\
\hline
\end{tabular}

Tableau 3 : Pronoms généralement accentués et non accentués

72 En opérant une synthèse des divers éléments observés dans le corpus, on note que l'emploi de formes pleines et l'accentuation paraissent remplir deux fonctions distinctes :

73 1) Formes pleines et accentuation permettent tout d'abord la désignation immédiate des entités extralinguistiques, d'où l'accentuation phrastique secondaire et la forme forte des mots lexicaux désignant des ensembles de propriétés ainsi que celles des pronoms démonstratifs, marqueurs de deixis. Cette remarque s'applique également aux pronoms interrogatifs et aux relatifs fusionnés, genrés et à la référentialité élevée.

2) Elles permettent également de signaler un degré plus faible d'accessibilité mentale. C'est le cas des formes fortis de IT, mais aussi de son accentuation emphatique, celle-ci signalant la nécessité de fournir un effort particulier pour accéder au référent. 
Cette remarque paraît aussi s'appliquer aux pronoms interrogatifs, marqueurs d'indéfinitude, ainsi qu'aux démonstratifs, dans la mesure où la deixis implique un degré d'accessibilité plus faible que l'anaphore stricto sensu (cf. par exemple Ariel 2000 ; Cornish 2003, 2006).

75 A l'inverse, l'emploi de formes faibles et l'absence d'accentuation signalent d'une part un faible degré de référentialité (ceci est particulièrement apparent dans le cas de THAT relatif et celui du pronom there) et la désignation indirecte des entités extralinguistiques par le biais d'opérations mentales telles que l'anaphore. Elles marquent d'autre part un degré plus élevé d'accessibilité du référent, ce qui apparaît très nettement dans le fonctionnement phonologique des pronoms personnels.

76 Nous formulons ici l'hypothèse que l'ensemble de ces facteurs peut être analysé en ne retenant que le critère de l'accessibilité. En effet, le gradient d'Ariel (2000) montre que moins un référent est accessible, plus la forme linguistique requise pour le désigner doit être substantielle. Lorsque rien n'a été dit d'un référent ou lorsque les éléments du contexte de communication sont trop faibles, diffus ou complexes pour permettre son identification immédiate, on comprend aisément qu'il soit nécessaire de le désigner très précisément, voire de le définir. Ceci implique l'emploi de formes linguistiques élaborées. ${ }^{32}$ Inversement, lorsque le référent se trouve au premier plan du discours, il suffit alors de rappeler sa présence, et une forme succincte suffit pour permettre sa représentation. ${ }^{33}$

77 Cette corrélation inverse entre degré d'accessibilité et élaboration des formes linguistiques ne concerne pas uniquement les formes graphiques, mais aussi le poids phonologique des formes linguistiques, et par conséquent leur éventuel degré de réduction ou d'accentuation, que celle-ci soit secondaire ou nucléaire.

78 En vertu de cette hypothèse, nous proposons d'expliquer l'accentuation phrastique secondaire des pronoms interrogatifs par le fait que ceux-ci apparaissent par définition dans un contexte où l'identité de leur référent n'a pas été révélée. Les pronoms interrogatifs ne fournissent en outre que peu d'indices relatifs aux caractéristiques de ce référent, puisqu'ils ne portent que la marque du genre - personne ou non personne.

C'est également ce peu d'indices sémantiques - à savoir le marquage du genre seul - qui pourrait permettre de rendre compte de l'accentuation systématique des relatifs fusionnés, malgré leur degré plus élevé de référentialité. Celle des démonstratifs s'expliquerait quant à elle par le signalement de l'opération de deixis, plus complexe que l'anaphore. Cette dernière opération est, pour ce qui la concerne, généralement marquée par des pronoms personnels non accentués.

80 Si cette intuition s'avérait exacte, l'absence de forme faible du pronom personnel de troisième personne du pluriel they pourrait ne pas être conditionnée exclusivement par la résistance des diphtongues à la réduction (cf. 1.1.5), mais aussi par le fait qu'il ne fournisse que relativement peu d'indices en vue de l'identification de son référent. Contrairement aux pronoms personnels de troisième personne du singulier, il ne porte en effet pas la marque du genre. D'autre part, à l'instar des pronoms démonstratifs, qui ne se réduisent pas, il marque une opération de deixis signalée par le morphème $\mathrm{TH}^{-34}$.

81 La non accentuation des pronoms personnels apparaît motivée par le fait que leur référent se trouve dans en règle générale au premier plan du discours. C'est lorsque ce n'est pas le cas qu'il devient nécessaire de recourir à l'emphase (cf. l'occurrence de IT 
accentué examinée en 2.8), voire à des procédés de compensation, comme le recours à des formes fortis (1.9).

Le pronom there, comme on l'a souligné au cours de l'analyse (1.8), apparaît entre autres dans des constructions qui gardent la mémoire d'énoncés où ce déictique locatif était adverbe de lieu. Dans les prédications d'existence, c'est la composante spatiale de la situation d'énonciation et, par métonymie, la situation elle-même, qui se trouve thématisée et, d'une certaine manière, présupposée. Dans ces conditions, le référent du pronom apparait éminemment accessible, et l'on s'explique facilement que there apparaisse fréquemment non accentué sous sa forme faible. Quant au relatif THAT - si toutefois l'on admet qu'il soit référentiel -, il apparait en principe immédiatement après le terme qui désigne son référent, d'où le haut degré d'accessibilité de ce dernier.

A partir des observations émises ici, il est donc possible de classer les pronoms du corpus en deux catégories : les pronoms faibles, qui ne reçoivent prototypiquement pas d'accentuation et sont fréquemment réduits, et les pronoms forts, prototypiquement accentués et sans forme faible (cf. tableau 4). Les pronoms faibles sont généralement employés pour désigner des référents facilement accessibles, alors que les pronoms forts le sont pour désigner des référents dont le degré d'accessibilité est moindre. Les pronoms indéfinis et réfléchis et indéfinis ne sont pas pris en compte dans ce tableau synthétique dans la mesure où leur polysyllabicité constitue une contrainte rythmique qui leur impose une accentuation phrastique secondaire.

\begin{tabular}{|c|c|}
\hline $\begin{array}{l}\text { Pronoms prototypiquement faibles } \\
<=>\text { référent au ler plan du discours }\end{array}$ & $\begin{array}{l}\text { Pronoms prototypiquement forts } \\
<=>\text { référent plus difficilement accessible }\end{array}$ \\
\hline $\begin{array}{l}\text { - Pronoms personnels } \\
\text { - Relatif THAT } \\
\text { - « Pronom » there dans les constructions } \\
\text { existentielles }\end{array}$ & $\begin{array}{l}\text { - Pronoms démonstratifs } \\
\text { - Pronoms relatifs « fusionnés » } \\
\text { - Pronoms interrogatifs }\end{array}$ \\
\hline
\end{tabular}

Tableau 4 : Classification des pronoms « faibles » et « forts »

\section{Conclusion}

L'examen des pronoms de notre corpus laisse fréquemment apparaître une correspondance entre le poids phonologique et la charge sémantique des formes observées. Ainsi, les pronoms démonstratifs et les relatifs « fusionnés ", qui permettent de désigner directement des entités du monde extralinguistiques et dont la référentialité évoque celle des syntagmes nominaux, apparaissent-ils plus susceptibles d'être produits sous leur forme forte, voire de recevoir systématiquement un accent phrastique secondaire, à l'instar des substantifs et des autres mots lexicaux. Les pronoms personnels, qui ne permettent de désigner des entités du monde extralinguistique que de manière indirecte, en marquant une opération d'anaphore, ne reçoivent en revanche pas prototypiquement d'accent phrastique secondaire et apparaissent le plus souvent sous leur forme faible. Le pronom there et le relatif THAT, dont le degré de référentialité est peu élevé, ont subi une désémantisation. En règle générale, ils ne reçoivent pas d'accent phrastique secondaire. There apparaît en outre éminemment susceptible d'être réduit alors que relatif тнат l'est systématiquement.

Parallèlement, il apparaît que les pronoms personnels, prototypiquement employés inaccentués et réduits pour désigner des entités situées au premier plan du discours, 
sont également susceptibles d'apparaitre sous des formes plus fortes, voire de recevoir un accent emphatique lorsque l'accessibilité de leur référent s'avère plus complexe.

Notre hypothèse est que ces deux phénomènes ne constituent que deux manifestations d'un même principe, à savoir le fait que le degré d'élaboration d'une forme linguistique est déterminé par celui de l'accessibilité de son référent. Un faible degré d'accessibilité contraint en effet à recourir à des formes élaborées, nécessaires à une désignation spécifique et précise, alors que la désignation d'un référent dont l'accessibilité est élevée ne requiert que des formes plus légères et succinctes. On trouve ainsi à une extrémité du gradient les groupes nominaux complexes, qui permettent de désigner des référents dont la représentation n'a pas même été activée, et à l'autre l'effacement total des formes signifiantes, susceptible de se produire lorsque le référent se trouve au tout premier plan du discours. Le poids phonologique des formes linguistiques apparait ainsi comme une composante à part entière de leur élaboration, le degré d'accentuation et de réduction de pronoms constituant des éléments de ce gradient au même titre que certains critères lexico-grammaticaux.

L'accentuation phrastique secondaire paraît alors renforcer le poids sémantique des mots lexicaux, permettant ainsi de remédier à la faible accessibilité de l'entité généralement dénotée par le syntagme dont ces derniers constituent le noyau. L'accentuation emphatique des pronoms personnels semble par contraste contrebalancer le peu de charge sémantique qui leur est associée dans les cas où l'accessibilité de leurs référents, ordinairement élevée, s'avère paradoxalement problématique. De manière comparable, nous suggérons que l'accentuation phrastique secondaire systématiquement appliquée aux relatifs fusionnés et aux pronoms interrogatifs permet de compenser le déficit sémantique occasionné par le fait que ces derniers ne portent que la marque du genre. Une situation comparable existe pour les pronoms démonstratifs, qui ne portent que la marque du nombre. On pourrait du reste envisager que l'absence de forme faible du pronom personnel de troisième personne they, non genré, soit motivée par un phénomène apparenté.

L'hypothèse que nous proposons ici synthétise et développe en grande partie les observations de Gundel et al. (1993) et Ariel (2000) en les appliquant de manière plus générale à domaine situé à l'interface de la syntaxe et de la sémantique. ${ }^{35}$ Elle met en outre en lumière le rôle d'éléments phonologiques tels que la réduction et le degré d'accentuation dans le marquage de l'accessibilité au même titre que les choix des opérateurs grammaticaux et des formes lexicales.

Il pourrait pour finir être utile d'approfondir la recherche sur le comportement des pronoms réfléchis et indéfinis, dont la polysyllabicité contraint le comportement rythmique, mais dont certains éléments se réduisent parfois. Le nombre limité d'occurrences présentes dans notre enregistrement ne nous semble pas susceptible de permettre l'identification des facteurs qui pourraient favoriser, voire contraindre le choix entre formes fortes et formes faibles, mais l'analyse d'un corpus plus étendu pourrait constituer un premier pas en vue de les définir. 


\section{BIBLIOGRAPHIE}

Ariel, M. (2000). The development of person agreement markers: From pronoun to higher accessibility markers. In Barlow, M. \& Kemmer, S. (eds.) Usage-Based Models of Language, 197-260. Stanford, CA: CSLI.

Auwera, J. van der. (1985). Relative that - a centennial dispute. Journal of Linguistics 21(1), 149-179.

Cornish, F. (1999). Anaphora, Discourse and Understanding. Oxford: Oxford University Press.

Cornish, F. (2003). The roles of (written) text and anaphor-type distribution in the construction of discourse. Text 23(1), 1-26.

Cornish, F. (2006). Relations de cohérence en discours : critères de reconnaissance, caractérisation et articulation cohésion-cohérence. CORELA HS-5.

URL: http://journals.openedition.org/corela/1456

Cotte, P. (2012). Genre et pronoms en anglais. Anglophonia/Sigma 16(32).

URL : http://journals.openedition.org/anglophonia/137

Cruttenden, A. (2001) Gimson's Pronunciation of English. 6th ed. London: Arnold.

Cruttenden, A. (2014) Gimson's Pronunciation of English. 7th ed. London: Routledge.

Davidse, K. (2000). A constructional approach to clefts. Linguistics 38, 1101-1131.

Di Cristo, A. (2003). De la métrique et du rythme de la parole ordinaire : l'exemple du français. Semen 16, Rythme de la prose.

URL : http://semen.revues.org/document2944.html

Gimson, A.C. (1965). An Introduction to the Pronunciation of English. Caxton Hill: Austin and Sons.

Gundel, J., Hedberg, N. \& Zacharski, R. (1993). Cognitive status and the form of referring expression in discourse. Language 69(2), 274-307.

Jones, D. (2011). Cambridge English Pronouncing Dictionary, 18th ed., ed. by P. Roach, J. Setter \& J. Esling. Cambridge: Cambridge University Press.

Khalifa, J.-C. (1999). La syntaxe anglaise aux concours. Théorie et pratique de l'énoncé complexe. Paris : Armand Colin.

Larrya, P. \& Rivière, C. (1999). Grammaire explicative de l'anglais. Harlow : Longman.

Roach, P. (2009). English Phonetics and Phonology. Cambridge: Cambridge University Press.

Suhamy, H. (2014). L'Accentuation dans le vers de Shakespeare. Actes des congrès de la Société française Shakespeare 31. URL : http://journals.openedition.org/shakespeare/2835

Wells, J. (1982). Accents of English. Vol. 1: An Introduction. Cambridge: Cambridge University Press. Wells, J. (2008). Longman Pronunciation Dictionary. 3rd ed. London: Longman.

Pages web / sources en ligne :

Dictionnaire Merriam-Webster : https://www.merriam-webster.com

Glossaire français-anglais de terminologie linguistique SIL : https://feglossary.sil.org/fr

Présentation du colloque TLD 6 sur le site de l'Université Grenoble-Alpes : 
https://www.univ-grenoble-alpes.fr/medias/fichier/presentation-et-programme-previsionneltld-6_1537190561894-docx. Consulté le 04 novembre 2019.

Trésor de la Langue Française informatisé (TLFi) : http://atilf.atilf.fr/

\section{NOTES}

\section{URL : http://atilf.atilf.fr/}

2. https://www.univ-grenoble-alpes.fr/medias/fichier/presentation-et-programmeprevisionnel-tld-6_1537190561894-docx

3. Les exemples donnés à analyser dans le texte initial sont indiqués par des chiffres entre parenthèses ; les autres exemples, tirés ou non du texte, sont indiqués par des chiffres entre crochets.

4. Cette « isochronie " n'est que relative (Roach 2009). Elle n'a pu être corroborée par aucune étude instrumentale (Cruttenden 2014). Comme précisé ici, cependant, la fréquence à laquelle les syllabes accentuées de l'anglais sont produites tend à être perçue comme régulière (Di Cristo 2003).

5. Nous utilisons ici les ensembles lexicaux (lexical sets) défini par Wells (1982) pour désigner les voyelles de l'anglais de manière non équivoque. Selon la tradition, on les représentera en petites majuscules. KIT représente ainsi la voyelle observée dans la réalisation d'unités lexicales telles que ship, sick, bridge, etc. Nous adoptons cette approche pour éviter de devoir choisir arbitrairement les composantes du système phonémique d'un accent spécifique (la $R P$, par exemple) plutôt qu'un autre pour servir de base de comparaison pour les autres accents.

6. Il existe en réalité plusieurs formes faibles car il existe divers degrés de réduction, le degré ultime de réduction pour un phonème vocalique isolé consistant en un schwa.

7. Ici encore, on note que plusieurs formes faibles de ce pronom sont répertoriées, ce qui achève de démontrer que la réduction est un phénomène graduel. Dans les syllabes qui comportent des consonnes, le degré ultime de réduction consiste en la disparition pure et simple de la voyelle, qui laisse alors place à une consonne syllabique. (you => /j/ dans un registre familier).

8. Ce type de variante est relativement fréquent en anglais américain, alors qu'en anglais britannique, elle est plus caractéristique d'un style relâché et de certains basilectes, notamment de l'accent cockney (cf. la transcription du parler d'Elisa Doolittle dans My Fair Lady).

9. C'est notamment la raison pour laquelle les modaux may et might, qui comportent des diphtongues, sont les seuls à ne pas avoir de forme faible.

10. Les pronoms indéfinis de l'anglais sont donc genrés puisqu'ils marquent la distinction entre la personne et la non-personne (cf. Cotte 2012).

11. On recense cependant des occurrences de forme faible de anything, anyone, anybody, principalement dans le énoncés négatifs ou interrogatifs.

12. Sauf, peut-être, pour ce qui est de this en anglais américain. Après avoir évoqué le fait que la faible /ðəs/ du déterminant this soit attestée dans certains tours figés (this afternoon, this evening, this morning), Wells (LPD, 2008) ajoute en effet : «In American English, this weak form is used more widely ». Ceci peut laisser supposer que dans les variétés américaines, la réduction est susceptible d'affecter non seulement le déterminant, mais encore le pronom démonstratif. La mention faite par Jones (CEPD, 2011) d'une forme faible décrite comme observée de manière occasionnelle ( occasional weak form ») concerne en premier lieu les tours également cités dans le LPD et paraît se référer au seul déterminant.)

13. Cruttenden ne fait pas ici explicitement mention de formes fortes ou de formes faibles, mais se réfère à l'accentuation systématique de certains mots dans la chaîne parlée. Cependant, comme nous l'avons vu lorsque nous avons défini la spécificité du rythme de l'anglais (cf. 
introduction), les mots systématiquement accentués ne possèdent normalement pas de forme faible. De telle sorte que la précision fournie ici implique que la raison pour laquelle les pronoms démonstratifs ne possèdent pas de forme faible est le fait qu'ils doivent être considérés comme des mots lexicaux.

14. <https://www.merriam-webster.com/dictionary/there>

15. Cette analyse de there en tant que vocatif n'est pas sans intérêt. Elle pose la question de sa réinterprétation dans des énoncés tels que Hi there, David!, dans lesquels il apparaît suivi d'un vocatif canonique.

16. La catégorie du nom et celle de l'adverbe peuvent de prime abord sembler assez distinctes, mais il existe entre elles des similitudes, voire des chevauchements non dénués d'intérêt, notamment dans le domaine de la localisation spatiale (cf. par exemple des énoncés comme Go west, young man! ou I am coming home).

17. There-cleft, cf. par exemple Davidse 2000.

18. La thématisation est un phénomène fréquent avec les compléments de lieu (cf. des énoncés comme Up went the balloon [cf. Larreya \& Rivière 1999] ou In the corner was a chair.)

19. Cf. Wilhelm (ce volume) pour une définition des termes fortis et lenis dans ce contexte particulier.

20. Utilisé dans l'acceptation que lui donne Cornish (1999, 2003, 2006), ce terme désigne ici la représentation mentale du référent.

21. Cette remarque s'applique aux pronoms personnels de l'anglais. Dans d'autres langues, comme le gallois, où le pronom personnel féminin de la troisième personne est employé pour désigner le temps chronologique et météorologique, elle pourrait être sujette à controverse.

22. Comme nous l'avons vu, ceci ne concerne pas les pronoms personnels (cf. 1.1.2).

23. Une variante de ce type est attestée dans le LPD.

24. On a choisi cette appellation dans la mesure où elle permet de marquer la dichotomie tonique / non tonique. Ceci ne veut pas dire qu'il n'existe pas une hiérarchie plus complexe entre les degrés d'accentuation des syllabes dans les énoncés.

25. Ou unité intonative.

26. Nous employons ici le terme emphatique pour désigner tout cas dans lequel un mot autre que le dernier élément lexical d'une unité intonative reçoit l'accent tonique. Ceci concerne principalement les situations suivantes: (i) usages conditionnés par la hiérarchie des informations en contexte de communication ; (ii) usages contrastifs ; (iii) usages de type métalinguistique ; (iv) usages dans certaines structures coordonnées. Le terme emphatique est parfois également employé pour se référer au signalement d'une force expressive particulière, y compris dans les cas de renforcement sémantique par expression du haut degré (cf. notes du colloque TLD sur l'exclamation). Pour éviter toute ambiguité, nous proposons de réserver le terme « emphatique » pour désigner les usages conditionnés par la hiérarchie des informations, contrastifs, métalinguistiques et en structure coordonnée, et de recourir à celui d'« expressif » pour nous référer à la deuxième situation évoquée ci-dessus. D'autres préfèrent alors la désignation d'«accent affectif» ou celle d'«accent d'expressivité». (Voir par exemple le glossaire de la SIL : <https://feglossary.sil.org/fr>.)

27. On trouve également dans l'enregistrement une occurrence de something porteur d'un accent phrastique secondaire: / I get a chance to give something BACK /. C'est ici la règle du dernier élément lexical qui s'applique et c'est l'adverbe back qui porte l'accent tonique.

28. On pourrait ainsi proposer la traduction suivante du premier de ces énoncés : «Vous pouvez faire en sorte qu'absolument n'importe qui puisse vous comprendre, pas vrai ? "

29. Par exemple: 'The woman whom I saw yesterday' really sounds too formal. Instead you should say 'The woman that I saw yesterday'.

30. L'absence d'accentuation de IT résulte en réalité du fait qu'il est habituellement utilisé en position proclitique ou enclitique (cf. par exemple Suhamy 2014 :17). 
31. Ces déclencheurs d'antécédents sont, à notre sens, non seulement les énoncés produits 11.32 (02'15”), 34 (02'15”), 44 (02'52”), 56 (03'43”), 64 (04'19”), mais encore divers éléments de la situation d'interaction: l'échange avec Al Gore (05'24" sqq.), utilisé par Tony Robbins pour illustrer son propos, divers éléments prosodiques tels que la fréquence mélodique, le volume sonore et la qualité de voix, et l'émotion manifestée par l'auditoire (cf. Wilhelm, ce volume).

32. Un substantif modifié par un groupe prépositionnel ou une proposition relative, par exemple. C'est précisément ce type de forme que l'on trouve au bas de l'échelle d'accessibilité d'Ariel (cf. fig. 1).

33. Cette forme linguistique est même susceptible de disparaître tout à fait, comme on le voit à l'extrémité supérieure de l'échelle d'Ariel (cf. fig. 1).

34. Nous sommes reconnaissant à Laurence Vincent-Durroux pour les suggestions qui se trouvent à l'origine de ce paragraphe.

35. Certains préféreront parler ici de composante pragmatique.

\section{RÉSUMÉS}

La catégorie des pronoms est éminemment difficile à circonscrire ; on reconnait usuellement l'existence de sous-catégories de pronoms assez dissemblables. À partir d'un corpus oral, nous examinons les caractéristiques phonétiques et phonologiques de différents types de pronoms utilisés en discours afin d'identifier des phénomènes communs à leurs emplois et de recenser leurs traits définitoires partagés. À travers l'examen de deux phénomènes corrélés mais distincts, à savoir la réduction et l'accentuation phrastique, nous mettons en évidence certains des liens qui existent entre forme phonétique et valeur sémantique des pronoms et dégageons de nos observations certains principes généraux. Postulant l'existence de deux types de pronoms : les pronoms phonologiquement faibles et les pronoms phonologiquement forts, nous suggérons en effet qu'il existe une relation étroite entre le poids phonétique et la référentialité des formes pronominales. Sur la base de nos observations, nous formulons également l'hypothèse qu'il existe en dernière analyse un rapport étroit entre la masse phonétique des pronoms et le degré d'accessibilité mentale de leur référent.

The category of pronouns, which admittedly includes several heterogeneous subcategories, is notoriously difficult to delineate. This corpus-based article examines the phonetic and phonological characteristics of various types of pronouns in a spoken discourse context in order to identify their shared definitional traits. Two intertwined though distinct phonological phenomena, i.e. reduction and sentence-level stress, enable us to highlight some of the links between the phonetic form of pronouns and their semantic value. We then extract a number of general principles from our observations. For instance, we posit the existence of two main phonological kinds of pronouns - strong and weak - and suggest that their phonetic weight is closely correlated to their level of referentiality. We also hypothesise that there ultimately exists a close link between the phonetic bulk of pronouns and the mental accessibility of their referents. 
INDEX

Mots-clés : Pronoms, phonétique, phonologie, poids phonétique, référentialité

Keywords : Pronouns, phonetics, phonology, phonetic weight, referentiality

\section{AUTEUR}

\section{STEPHAN WILHELM}

Université de Bourgogne - Laboratoire TIL (Texte, Image, Langage) 